\title{
¿QUIÉNES SOMOS NOSOTRAS? ASPECTOS FRONTERIZOS DEL FEMINISMO EN REALIDADES LATINOAMERICANAS
}

\author{
RESUMEN
}

Lourdes C. Pacheco Ladrón de Guevara

pacheco11@yahoo.com

Universidad Autónoma de Nayarit,Nayarit, México.
El presente texto discute las categorías desarrolladas por el feminismo y los estudios de género para explicar la condición de las mujeres y los coloca en tensión respecto de las realidades de las mujeres afrodescendientes e indígenas de América Latina. Con ello se pretende mostrar las fronteras de las categorías con la finalidad de avanzar en los estudios a partir de contextos históricos específicos que permitan otorgar nuevas conexiones entre la teoría y las realidades de las mujeres latinoamericanas.

PALABRAS CLAVE: Feminismo. Género. Mujeres.

\section{QUEM SOMOS NÓS? ASPECTOS FRONTEIRIÇOS DO FEMINISMO EM REALIDADES LATINOAMERICANAS}

\section{RESUMO}

O presente texto discute as categorias desenvolvidas pelo feminismo e pelos estudos de gênero para explicar a condição das mulheres e confronta-las com as realidades das mulheres afrodescendentes e indígenas da América Latina. Com ele se pretende mostrar as fronteiras das categorias com a finalidade de avançar nos estudos a partir de contextos históricos e específicos que permitam estabelecer novas conexões entre a teoria e as realidades das mulheres latino-americanas.

PALAVRAS-CHAVE: Feminismo. Gênero. Mulheres. 


\section{INTRODUCIÓN}

Tanto el feminismo como los estudios de género han promovido transformaciones en el camino hacia la igualdad de las mujeres y la eliminación de las prácticas que limitaban su autonomía e impedían el ejercicio de su libertad. Los aportes del feminismo entendido como "toda teoría, pensamiento y práctica social, política y jurídica que tiene por objetivo hacer evidente y terminar con la situación de opresión que soportan las mujeres y lograr así una sociedad más justa que reconozca y garantice la igualdad plena y efectiva de todos los seres humanos" (AGUILERA, 2009, p. 46) se encuentra en el centro mismo de la revolución democrática de la modernidad.

El concepto de género ha sido desarrollada con la intención de teorizar la construcción social y simbólica de la diferencia sexual (LAMAS, 2000), cuestionar el determinismo biológico y analizar la organización social entre mujeres y hombres (HAWKESWORTH, 1999). En este sentido ha abierto el estudio a diversos ámbitos más allá de las relaciones entre hombres y mujeres para encontrar intersecciones entre el poder, el sistema jurídico, la vida privada, el sistema económico, etc. A partir de ello ha sido posible ampliar la mirada en el sentido de que el sexo estructura prácticas y símbolos de la sociedad que impactan directamente en la detentación del poder (SCOTT, 1992).

Si bien el feminismo como teoría y práctica política de transformación y la categoría conceptual de género han abierto posibilidades epistemológicas de repensar el lugar que tienen las mujeres en la sociedad y abrir las posibilidades a nuevos planteamientos, la irrupción de la diferencia al interior de las mujeres, ha dejado al descubierto asignaturas pendientes: reconocer la diversidad de las mujeres, la colocación de quien enuncia, los puntos de partida. De ahí entonces que es dable preguntar ¿cuándo se habla de las mujeres, de qué se habla? ¿Quiénes somos nosotras? Ello incorpora el tema del feminismo y el género desde realidades latinoamericanas, de ahí que el presente artículo se proponga discutir aspectos fronterizos de es a vinculación.

\section{ARGUMENTACIONES: LAS PROPUESTAS DEL FEMINISMO}

El feminismo se constituyó como la posibilidad de cumplir con el proyecto igualitario de la llustración puesto que puso en evidencia la falta de cumplimiento de sus postulados en el caso de las mujeres. La llustración consecuente (CASTELLS, 1996) pretendía evidenciar las contradicciones del discurso ilustrado. Para ello, la teoría de los derechos humanos permitió establecer un corpus de ideas donde se sustentara la vindicación de la igualdad, el uso de la razón y el abandono de los prejuicios para las mujeres (SÁNCHEZ, 2005). El paso siguiente fue construir a la mujer como sujeto de derecho y por lo tanto, titular de los derechos reconocidos a los varones. En particular, la elección de su propio destino fuera del sometimiento en que se encontraba, el reconocimiento de derechos concretos como el derecho a la educación, al trabajo y a la ciudadanía.

El reconocimiento de los derechos de las mujeres así como la historia de la vindicación se realizó en Europa y en Estados Unidos con ritmos desiguales puesto que en este último país el movimiento por el reconocimiento de los derechos de las mujeres se convirtió en un movimiento social al vincularse al movimiento 
abolicionista y de reforma moral. En Europa en cambio, no fue un movimiento social sino que fueron argumentaciones de personas destacadas las que sustentaron la necesidad de ampliar los derechos de la llustración a las mujeres. Por ejemplo, John Stuart Mill presentó en 1866 la primera petición a favor del voto femenino (DE MIGUEL, 2000). Posteriormente, la emancipación de las mujeres europeas se vinculó al socialismo y por ende, se vinculó a la clase obrera.

Tanto en Europa como en Estados Unidos el feminismo tuvo como protagonistas principales a mujeres de las clases medias ilustradas, las cuales reflexionaron sobre sus propias condiciones de vida. Esta condición se convirtió en el eje estructurante de las teorías feministas.

\section{LOS UNIVERSALES DEL FEMINISMO}

La teoría feminista del reconocimiento de derechos dio lugar a estudios filosóficos, antropológicos, etc., que intentaron explicar el origen de la desigualdad de las mujeres, la subordinación, la falta de poder, etc. Entre las propuestas teóricas que han dado sustento a las teorías feministas se encuentran los conceptos de patriarcado, el sistema sexo género y el equiparamiento de la mujer a la naturaleza.

La mujer como naturaleza tuvo su fundamento en los ciclos biológicos del cuerpo de las mujeres, en particular, en la menstruación. La menstruación se convirtió en la diferencia fundamental entre el cuerpo del hombre y de la mujer. Los ciclos de las mujeres han sido asumidos por las distintas culturas como ciclos naturales. En consecuencia, vincular lo natural a lo primitivo permitió encasillar a las mujeres como amenaza a lo civilizatorio, se le adjudicaban atributos de esterilidad y peligrosidad ya que

[...] impedía germinar los cereales y agriaba los mostos; por su contacto morían las hierbas, los árboles perdían sus frutos, el hierro era atacado por el orillo y los objetos de bronce se ennegrecían; los perros que la hubieran sorbido contraían la rabia (DE AQUINO, 1975, p. 65)

\section{TRABALHO E DIVISÃO SEXUAL DO TRABALHO}

A partir de ello, se excluyó a las mujeres de lo divino, posteriormente relacionado con la ciudad, lo culto, lo civilizado. Al no formar parte de lo sagradocivilizado, las mujeres pertenecían a un mundo aparte, el mundo de la naturaliza donde se realiza lo indómito, lo salvaje, lo que agrede lo social-humanodivino.Si la naturaleza representa el mundo originario del cual se desprendió la cultura, se convierte en un ámbito que atenta sobre lo humano por lo que debe ser controlado, dominado. La mujer pertenece a ese ámbito y permanece en él.

Las mujeres como naturaleza tiene también la vertiente de lo contemplado: En el Diario del seductor, Kierkegaard dice:

La esencia de la mujer viene indicada justamente como gracia, expresión que nos recuerda la vida vegetativa; ella es como una flor, gusta decir a los poetas, y, por último, lo que en ella hay de espiritual tiene algo de vegetativo. Ella entra en los límites de la naturaleza y es, 
por esto, libra más bien estéticamente. En un sentido más profundo, es liberada por medio del hombre (AMORÓs, 1985, p. 162).

\section{LAS FRONTERAS DE LAS CATEGORÍAS}

La teorización acerca del género inició en la década de los setenta a partir de los estudios de las condiciones de las mujeres lo que permitió explorar la condición de las mujeres más allá del determinismo biológico, enfatizó en las condiciones de las mujeres como producto de la socialización, las prácticas disciplinarias y culturales (BUTLER, 2001). El concepto de género planteó que cualquier cambio en una de las partes afectará también a la otra por lo que se extrapoló el concepto a toda la sociedad.

En las argumentaciones sobre la vigencia del concepto, Joan Scott (1997, p. 170)propone que el género es una categoría útil porque "[...] proporciona una manera de decodificar el significado y de entender las conexiones complejas entre varias formas de interacción humana" por lo que en lugar de extenderlo como una categoría universal debe realizarse una auténtica historización. En lugar de aplicar el género como una categoría ahistórica, debe analizarse la forma como se conformaron las sociedades a fin de encontrar por qué ocurrieron.

Sandra Harding (1996, p. 117) reconoce que el género aparece sólo en formas culturales específicas pero ello no le resta capacidad de análisis:

La vida social determinada por el género se produce a través de três procesos distintos: es el resultado de asignar metáforas de género dualistas a varias dicotomías percibidas que rara vez tienen algo que ver com diferencias de sexo (simbolismo de género); es la consecuencia de recurrir a esos dualismos de género para organizar la actividad social, dividiendo actividades sociales necesarias entre diferentes grupos de humanos (estructura de género); es una forma de identidad individual social mente construida, sólo imperfectamente correlacionada con la realidad o con la percepción de las diferencias de sexo (género individual).

La defensa del género de Scott (1997) y Harding (1996) refuerzan la importancia que la estructura de género y el simbolismo de género han tenido para criticar identidades esencializadas y crean la posibilidad de crear políticas que atraviesen las condiciones de raza, etnia, orientación sexual y clase social, por lo que confieren una posibilidad de vinculación entre la identidad, la subjetividad, la conexión de la psique, la organización social, la experiencia del cuerpo, la sexualidad, la normatividad, etc.

Actualmente, las principales críticas al concepto estriban en la constatación de que el género siempre está mediado por la raza, la etnia, el color, la preferencia sexual y la clase social, de ahí que las teóricas del género hayan privilegiado solamente una opresión, la de la opresión patriarcal. Además de ello, el concepto partía del cuerpo sexuado de las mujeres, pero en la postmodernidad se ha puesto de manifiesto el cuerpo, el sexo y la sexualidad como histórica, cultural y socialmente construido (BUTLER, 2001), por lo que la categoría habría perdido la fuerza analítica que presentó en los últimos veinte años del siglo XX. 


\section{LA DECONSTRUCCIÓN DEL FEMINISMO OCCIDENTAL}

El feminismo denunció el lugar privilegiado del sujeto varón en la enunciación del mundo, la construcción de la epistemología científica, la normatividad, etc. Pero así como el feminismo se encargó de develar la falacia universal escondida en la parcialidad de los hombres blancos, adultos, heterosexuales y letrados, entre otras características, el feminismo negro se encargó de mostrar las limitaciones del feminismo occidental cuyo punto de partida fueron las mujeres blancas heterosexuales de clase media, que elevaban su propia condición a un universal de mujeres.

La adopción incondicional del feminismo occidental por parte de mujeres africanas presentaba varios problemas: por una parte dejaba de lado el análisis de las causas de la situación de desventaja de las africanas $y$, em segundo lugar, presentaba al hombre africano como el opresor y causante de las desigualdades existentes... al adoptar una perspectiva ajena a su propia cultura se estaba animando a la mujer africana a rechazar su papel tradicional en la familia. (OBIANUJU, 1995, p. 80-82)

Las luchas de las mujeres indígenas de América Latina, por su parte, dejaron al descubierto la necesaria intersección entre el feminismo, el género y la etnia, al menos en las discusiones sobre los derechos de las mujeres de los pueblos originarios ya que el establecimiento de las diferencias (de género, de condición étnica, de clase social, de religión, entres otras) son relaciones emanadas de construcciones histórico-culturales que no desaparecen cuando los distintos grupos culturales interactúan en un espacio pensado desde la homogeneidad cultural. Sin embargo, estas relaciones pueden cambiar al imprimirse en ellas nuevas consideraciones y reconocimiento de la otredad que valoren la cultura, las experiencias de vida de los distintos grupos culturales y los excluidos y se ponga en tela de juicio la homogeneidad cultural de los grupos dominantes.

En el proceso de deconstrucción del feminismo occidental, Maria Lugones (2008) afirma que el sistema categorial desarrollado por el feminismo y centrado en el concepto género encubre el origen colonial del sistema de género, puesto que las mujeres negras, afrodescendientes e indígenas fueron el objeto de la civilización y no sujetos.

El género que forma el centro del debate y prácticas feministas hegemónicas es una imposición y producción colonial. Mientras que los que la colonización produce como blancos burgueses son... las gentes a colonizar, domar, explotar de formas brutales, no tienen género en tanto no son propiamente humanos (LUGONES, 2008, p.78)

El género que forma el centro del debate y prácticas feministas hegemónicas es una imposición y producción colonial. Mientras que los que la colonización produce como blancos burgueses son... las gentes a colonizar, domar, explotar de formas brutales, no tienen género en tanto no son propiamente humanos (LUGONES, 2008, p.78)

En América Latina, las principales discusiones se han dado a partir del feminismo negro latinoamericano y de las reivindicaciones de las mujeres 
indígenas. El feminismo negro latinoamericano afirma que la "perspectiva feminista es diferente desde una vivencia etno-racial, afrodescendiente que desde una vivencia etno-racial dominante, generalmente blanca" (CAMPBELL: 2002). Las diferencias de la realidad histórica de las mujeres afrodescendientes en relación con las mujeres latinoamericanas se pueden centrar en lo siguiente:

- Las mujeres esclavas constituían parte de la fuerza productiva por lo que siempre fueron parte de la fuerza laboral. Consecuentemente, no estuvieron recluidas en el hogar

- Las mujeres esclavas laboraban en las mismas condiciones de los esclavos varones por lo que compartieron el ámbito de trabajo, sus condiciones, explotaciones y retribuciones

A las mujeres esclavas no se les permitía tener familia propia por lo que no participaron de la división de roles de género entre roles femeninos y masculinos

- Las mujeres esclavas eran separadas de sus hijos ya que los hijos de las mujeres esclavas eran considerados bienes del dueño esclavista. Ello impidió la construcción de lazos afectivos familiares

- Una vez terminada la esclavitud, las mujeres afrodescendientes continuaron en la vida laboral de la misma manera que los hombres A su vez, a partir del grupo étnico wixaritar1 las características de la realidad de las mujeres de los pueblos originarios son:

- Las mujeres wixaritari participan en el ámbito productivo de las comunidades indígenas, por lo que no ocurre la división del trabajo en actividades productivas y reproductivas.

- Las mujeres wixaritari se encuentran socializadas dentro de simbologías donde lo masculino y lo femenino comparten características en planos simétricos. Por ejemplo, de acuerdo a la cosmogonía del grupo, en la creación del mundo participaron madres y padres del panteón wixaritari: la Abuela Nakawue se considera la creadora de todo lo existente, en tanto que el Abuelo Tayao simboliza el fuego (ZING, 2000).

- Las mujeres wixaritari realizan la reproducción biológica y social de manera colectiva ya que las mujeres de una generación socializan a los hijos de todas las mujeres de esa misma generación, sin que exista una individualización de las actividades reproductivas. Las condiciones señaladas tanto de las mujeres afroamericanas como de las mujeres wixaritari, (características que pueden estar presentes en otros grupos indígenas de América Latina), marcan diferencias importantes con el resto de mujeres latinoamericanas, en particular:

- En ambos grupos no existió división del trabajo por sexos de manera antagónica como en la cosmovisión occidental

- En ambos grupos las mujeres no fueron recluidas al trabajo doméstico ni éste fue considerado inferior al trabajo productivo. En el caso de las mujeres wixaritari, el trabajo doméstico se realiza en el exterior de las viviendas por lo que no tiene el estigma de una actividad realizada en espacios cerrados, ocultos o desvalorizados. - Las mujeres afrodescendientes y wixaritari no transitaron del no trabajo al trabajo sino que siempre estuvieron en el trabajo productivo. Para la sobrevivencia de los grupos indígenas el trabajo de las mujeres es vital puesto que crean bienes de consumo agrícola, realizan recolección y cría de animales, seleccionan el maíz para la siembra, actividades que en conjunto contribuyen a la realización comunitaria del grupo. 
- En las mujeres wixaritari existe una culturización positiva de las mujeres que les otorga poder simbólico más allá de la mera actividad biológica de madre. Son símbolos de sabiduría y poder.

\section{LA DOMINACIÓN DE LA NATURALEZA}

Además de lo anterior, el feminismo desarrolló la idea de la inferiorización de las mujeres como un proceso histórico dentro del pensamiento heredado de los griegos para quienes la mujer era un hombre incompleto (ARISTÓTELES, 1990, p.574). Ello fue la base de la pretensión de la modernidad por controlar la naturaleza y convertirla en recurso productivo, la mujer, entonces fue considerada cercana a la naturaleza (alejada de la cultura) por compartir con ella procesos incontrolables. Sin embargo, el pensamiento de los pueblos indios no realiza la división de lo humano con la naturaleza, por el contrario, lo humano es parte de lo natural dado y en ello participan tanto los varones como las mujeres.

Por lo tanto, si en el pensamiento wixaritari no se desarrolló la pretensión de someter a la naturaleza, dominarla y controlar sus ciclos, sino por el contrario, "la naturaleza es la casa donde vivimos, el lugar que crearon los Antepasados" (BENITEZ, 2001). En la cosmogonía wixarika, lo humano es parte de la naturaleza y está poblada por lo sagrado, donde los wixaritari son unos más en el conjunto de los seres. El espíritu del agua, del viento y de la tierra; de las plantas y las estrellas; del mundo atisbado y del mundo presentido.

Para el grupo wixaritari todo lo existente es hermanado en un mismo nivel, lo mismo la hormiga que el tlacuache, el río que la espuma del mar. Los seresanimales tuvieron un protagonismo esencial para convertir el mundo en la posibilidad humana y hoy se refieren a ellos como personas-animales o personassemillas, los incorporan en la jerarquía parental como bisabuelos, abuelos, padres, madres, hermanos. No sólo son sus iguales, sino que son los que posibilitaron lo humano existente, de ahí que no se haya desarrollado el deseo de controlar, sino de participar con ellos en la creación continua de lo humano2.

En este sentido resalten las acotaciones de Sherry Ortner (2006) en poner en tensión la oposición naturaleza-cultura que fue la base del feminismo occidental en cuanto a discutir: 1) la universalidad de la dominación masculina; 2) la intencionalidad de la dominación masculina, 3) las distintas concepciones de naturaleza presentes en distintos grupos culturales.

Respecto a la universalidad de la dominación masculina la autora hace referencia a casos de sociedades donde los elementos de dominación masculina no son exactamente centrales para asegurar la superioridad masculina, de tal manera que la autora llega a afirmar la existencia de una cierta "igualdad de género" que podría extenderse a ciertas prácticas del grupo wixaritari señalado.

En cuanto a la intencionalidad de la dominación masculina Ortner cuestiona si ésta constituyó la "histórica derrota mundial de las mujeres" como lo señaló Federico Engels (1984) o si es una consecuencia del lugar social que fueron teniendo los hombres al ocuparse menos de la vida doméstica y más del ámbito de la cultura. Sin embargo, en cualquiera de los dos argumentos, la intencionalidad del dominio o las funciones desarrolladas por los varones, da por resultado sociedades donde los varones tienen un lugar preeminente. 
En la oposición naturaleza-cultura se cuestiona si esa relación dicotómica ocurre en todos los grupos culturales con el mismo valor asignado a la cultura y a la naturaleza o si corresponde sólo a la sociedad occidental. De ahí que en las sociedades donde la relación sociedad-naturaleza no se plantea en términos de superioridad o dominio, la dicotomía no alcanza a explicar la relación entre hombres y mujeres.

\section{CONSIDERACIONES FINALES}

El feminismo creó causales universales (como el patriarcado y el equiparamiento de la mujer a la naturaleza) con lo cual propuso explicaciones totalizadoras válidas para todas las sociedades y condiciones. En tanto que la utilización del género tiende a historizar los procesos, estudiar el cómo, para explicar el por qué.

Dentro de la argumentación anterior, queda por discutir entonces ¿quiénes somos nosotras? ¿a qué mujeres se refieren y toman como punto de partida tanto el feminismo como los estudios de género? Queda claro que tanto el feminismo como los estudios de género son esfuerzos teóricos y de acción práctica, social y política para transformar la desigual en que se encuentran las mujeres, esfuerzos realizados desde un cierto sujeto femenino ubicado en Europa y Estados Unidos.

La descentralización del sujeto mujer (blanca, heterosexual, de clase media) de la teoría feminista impacta en la propia construcción de la teoría y en el uso de conceptos categoriales como el género. Se convierte en un desafío a la propia teoría y a la vinculación con otras categorías como la raza y la etnia ya que tanto el género como la raza y la etnia forjan no sólo la identidad sino también las relaciones sociales y de poder. El impacto es mayor si se toma en cuenta que influye en la epistemología y metodología feminista.

El feminismo y los estudios de género están en deuda con las realidades de las mujeres afrodescendientes y de los pueblos originarios de América Latina puesto que las condiciones históricas en que han existido deben tomarse en cuenta dentro de las teorizaciones feministas y de estudios de género. En todo caso, los principios en los que se asienta la teoría feminista: la dominación universal masculina y la contraposición naturaleza-cultura, deben ser puestas en tensión en las realidades latinoamericanas. 


\title{
WHO ARE WE? FRONTIER ASPECTS OF FEMINISM IN REALITIES LATIN AMERICAN
}

\begin{abstract}
This paper discusses the categories developed by feminism and gender studies to explain the status of women and places them in tension with the realities of African descent and indigenous women in Latin America. This is to show the boundaries of the categories in order to advance in studies from specific historical contexts that enable issuing new connections between theory and reality of Latin American women.
\end{abstract}

KEYWORDS: Feminism. Gender. Women. 


\section{NOTAS}

${ }^{1}$ El grupo wixaritari o huichol habita la Sierra Madre Occidental en los estados de Nayarit, Jalisco y Zacatecas.

${ }^{2}$ Un ejemplo de ello es la negación de los witaritari por aplicar agroquímicos en sus parcelas debido a que envenenan a la madrecita Oliánaka, la Madre Tierra (Carrillo, 2011).

\section{REFERÊNCIAS}

AMORÓS, Celia. Crítica de la razón patriarcal.Barcelona: Antropos, 1985.

ARISTÓTELES. Historia de los animales. Madrid: Akal/clásica, 1990.

BENITEZ, José. Entrevista a marakame huichol. Zitakua, Nayarit, 2001.

BUTLER, Judith. El género en disputa: el feminismo y la subversión de la identidad. Programa Universitario de estudios de género. UNAM. Paidos, México, 2001.

CAMPBELL, Epsy. Una perspectiva sobre feminismo negro latinoamericano. In: Poderes cuestionado: sexismo y racismo en América Latina. CAREAGA, Gloria;

CAMPBELL, Epsy (coord.), Red de mujeres afrocaribeñas y afrolatinoamericanas, PUEG-UNAM, San José de Costa Rica, p. 72-78, 2002.

CARRILLO, Rutilio. Entrevista a wixaritari, Zitakua, Nayarit, 2011.

CASTELLS, Carme. Perspectivas feministas en teoría política. Barcelona: Paidós, 1996.

HARDING, Sandra. Ciencia y Feminismo, Morata, España, 1996.

DE AQUINO, Santo Thomás. La Summa Teológica, Porrúa, México, 1975.

AGUILERA, Samara De las Heras. "Una aproximación a las teorías feministas", en Revista Universitas, Madrid, n. 9, p. 45-82, 2009.

DE MIGUEL, Ana. Los Feminismos. In: AMORÓS, Celia (coord.). Diez palabras clave sobre lamujer, Estella: Editorial Verbo Divino, p. 2 a 5, 2000.

ENGELS, Federico. El origen de la familia, la propiedad privada y el Estado. Ciudad de Mexico: Ediciones Quinto Sol, 1984.

HAWKESWORTH, Mary. Confundir el género. Debate Feminista, Ciudad de México. n. 10, 1999. Disponível em:

www.debatefeminista.pueg.unam.mx/wpcontent/.../020_01.pdf. Acesso em: 20/01/2012. 
LAMAS, Marta. La construcción cultural de la diferencia sexual. Ciudad de México: PUEG-Porrúa, 2000.

LUGONES, María. Colonialidad y género. Tabula Rasa. Bogotá, n. 9, jul-dez, 73$101,2008$.

OBIANUJU ACHOLONU, Catherine. Motherism: The Afrocentric Alternative to Feminism. Owerri: Afa Publications, 1995.

ORTNER, Sherry. Entonces, ces la mujer al hombre lo que la naturaleza a la cultura?, AIBR, Revista de Antropología Iberoamericana. Disponível em: http://www.aibr.org/antropologia/01v01/articulos/010101.pdf. Acesso em: 20/01/2012.

SÁNCHEZ MUÑOZ, Cristina. Genealogía de la vindicación. In: BELTRÁN, Elena;

MAQUIEIRA, Virginia (Eds.). Feminismos. Debates teóricos contemporáneos. Madrid: Alianza Editorial, 2005.

SCOTT, Joan. 1997. Igualdad versus diferencia: los usos de la teoría postestructuralista. Debate Feminista, Ciudad de México, n. 5, 1997.Disponível em: www.debatefeminista.cieg.unam.mx/wp-content/.../005_09.pdf. Acesso em: 20/01/2012.

TRIMIÑO VELAZQUEZ, Celina de Jesús. Una mirada al feminismo en América Latina y el Caribe. Interrelación con el movimiento de mujeres. Aportaciones de la teología de la liberación a los derechos humanos, Universidad Carlos III de Madrid, Barcelona, p. 59-90, 2008.

ZING, Robert. Los huicholes. Instituto Nacional Indigenista, México, 2000.

Recebido: 27 de janeiro de 2013.

Aprovado: 09 de junho de 2017.

Como citar:

DE GUEVARA, Lourdes C. Pacheco Ladrón. ¿Quiénes somos nosotras? Aspectos

fronterizos del feminismo en realidades latinoamericanas. Cad. Gên. Tecnol., Curitiba, v.

10, n. 35, p. 58-68, jan.jun. 2017.

Correspondência:

Lourdes C. Pacheco Ladrón de Guevara

Universidad Autónoma de Nayarit, Ciudad de la Cultura "Amado Nervo"

63190 Tepic, Nayarit, México.

Direito autoral:

Este artigo está licenciado sob os termos da Licença Creative Commons-Atribuição 4.0 Internacional.

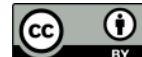

